Document downloaded from:

http://hdl.handle.net/10251/121351

This paper must be cited as:

Marqués-Villarroya, D.; Canós Marín, AJ.; Penaranda-Foix, FL.; García-Baños, B.; Catalá Civera, JM. (2018). Full-Wave Modal Analysis of a Novel Dielectrometer for Accurate Measurement of Complex Permittivity of High-Loss Liquids at Microwave Frequencies. IEEE Transactions on Microwave Theory and Techniques. 66(12):5760-5770.

https://doi.org/10.1109/TMTT.2018.2881136

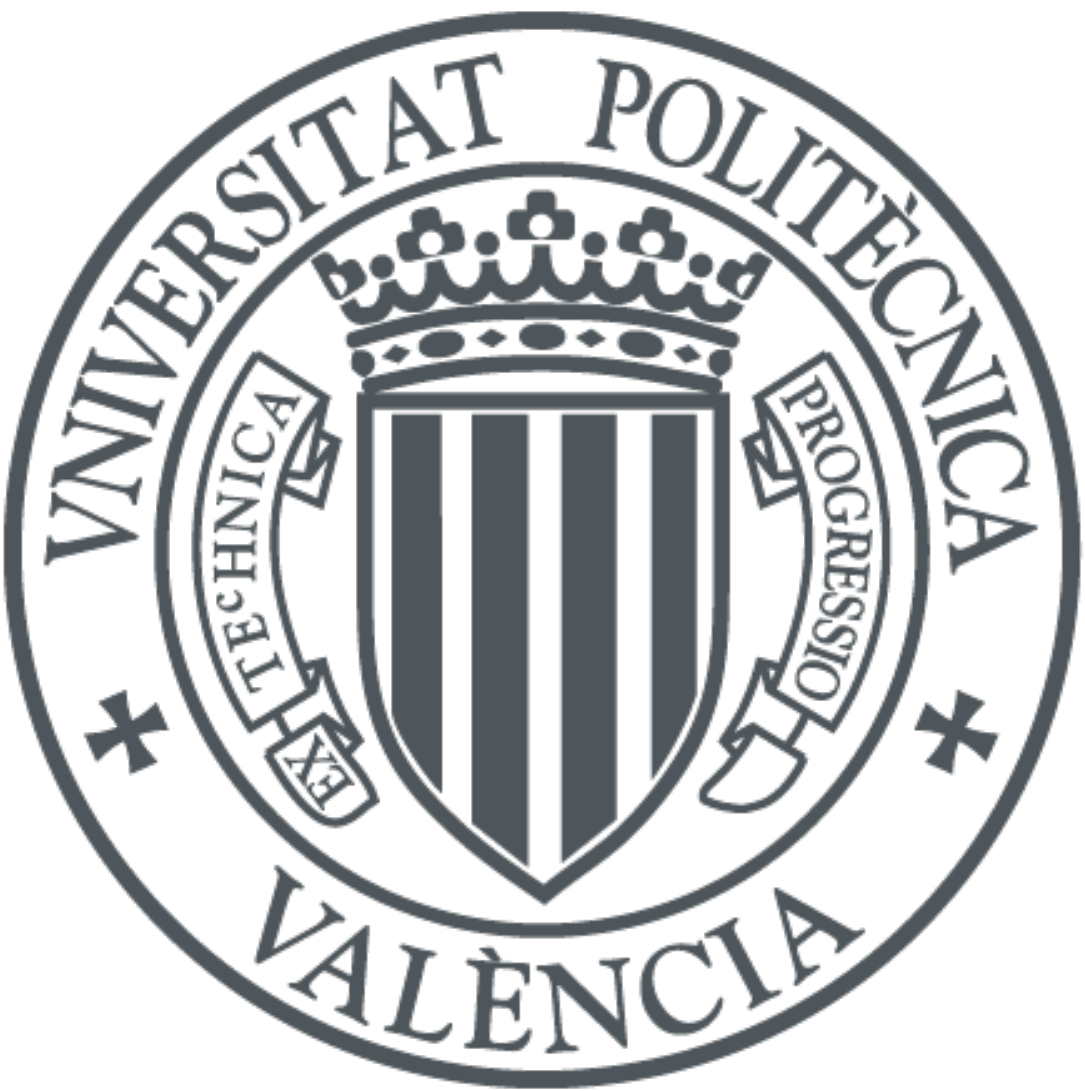

The final publication is available at

http://doi.org/ 10.1109/TMTT.2018.2881136

Copyright Institute of Electrical and Electronics Engineers

Additional Information 


\title{
Full-Wave Modal Analysis of a Novel Dielectrometer for Accurate Measurement of Complex Permittivity of High Loss Liquids at Microwave Frequencies
}

\author{
David Marques-Villarroya, Antoni J. Canós, Felipe L. Peñaranda-Foix, Senior Member, IEEE, Beatriz \\ Garcia-Baños, Jose M. Catala-Civera, Senior Member, IEEE
}

\begin{abstract}
A novel dielectrometer to measure the complex permittivity of high loss liquids is presented. The geometry consists of a reentrant cavity with insertion holes where the holder filled with liquid can be introduced readily. Radii of the insertion holes are large enough for a convenient pouring of the liquid. The electromagnetic analysis has been performed by a mixed modematching and circuit technique with the purpose of taking the high accuracy and fast convergence of the mode-matching and the flexibility and versatility of the circuit method. The convergence of the method is studied, and a procedure to estimate the result for an infinite number of modes is proposed. A calibration procedure is presented to minimize the error introduced by the numerical method. Mode charts are shown to analyze the behavior of resonant parameters for every mode. Some reference liquids are measured at different resonant frequencies and results are compared with those provided by other models of the literature.
\end{abstract}

Index Terms_-Permittivity, High Loss Liquids, mode-matching, full-wave modeling, Reentrant Cavity.

\section{INTRODUCTION}

$I^{2}$ NTEREST in dielectric characterization of polar liquids at Imicrowave frequencies has been increasing in recent last years because many applications derive from it, such as molecular characterization, dielectric heating, remote sensing, creation of tissue equivalent-materials or reference liquid standards, and design of calibration systems [1]-[3].

Many successful techniques have been developed to measure liquids' permittivity at microwave frequencies. First, openended coaxial probes were employed because their ease of use and their broadband characterization [4]-[5]. Later, in order to improve the accuracy of the measurements, transmission line techniques were developed, which are suitable to measure lossy materials (most of the liquids) at discrete frequencies [6]-[7]. Then, instead of transmission technique, better accuracy can be

Manuscript received May, 2018.

This work has been financially supported by "Programa de Ayudas de Investigación y Desarrollo (PAID) de la Universitat Politècnica de València”. Results are obtained under the project TEC2012-37532-C02-01 financed by Ministerio de Economía y Competitividad (MINECO) -Spanish Governmentand co-funded by ERDF (European Regional Development Funds) of European Union.

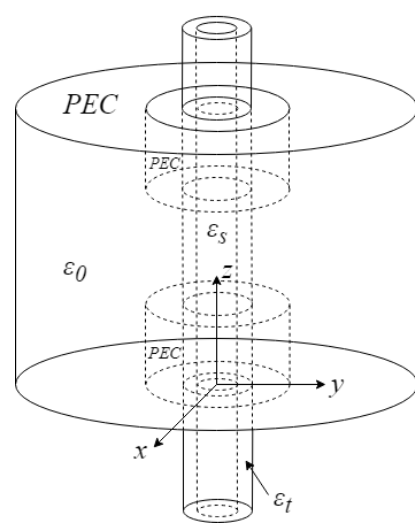

(a)

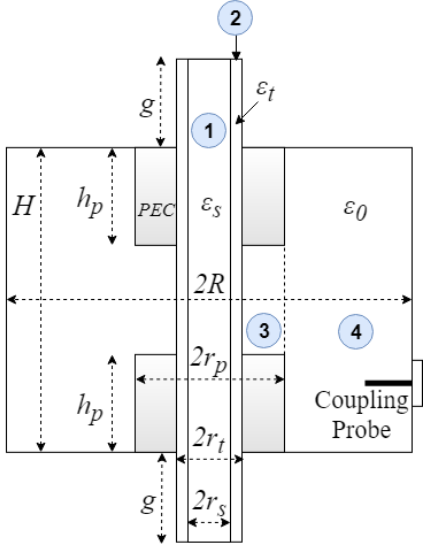

(b)
Fig. 1. Geometry of the analyzed cavity. (a) 3D view (b) Side view with dimensions.

obtained with resonant cells, since frequency measurements are more robust [8]. Although resonant techniques are typically used for low-loss materials, they can be used also for lossy materials if the cell provides a measurable quality factor. In fact, there are many configurations designed to measure liquids at microwave frequencies with resonant cells [9]-[17]. However, the measurement of high-loss liquids presents special difficulty in resonant cells. Only little amount of these materials can be placed inside the cell to obtain a measurable quality factor. Specially interesting is the work performed in [15], where the complex permittivity of high loss liquids is measured with resonant cylindrical cavities using symmetric modes $\mathrm{TM}_{0 \mathrm{np}}$ and $\mathrm{TE}_{0 \mathrm{np}}$, and the quality factor is improved using low loss filling materials. In this sense, in last years, dielectric characterization of lossy liquids has been focused on microfluidic sensors [18][21], which use a very small sample size, making difficult the sample preparation process. The cavity cell proposed in this

This paper is an expanded version from the 2018 International Microwave Symposium, Philadelphia, PA, USA, 10-15 June 2018

Authors are with Instituto ITACA, Universitat Politècnica de València, Camino de Vera, s/n, 46022-Valencia, Spain (David Marques Villarroya, the corresponding author, phone: +34-96 387 9742; e-mail: damarvi3@itaca.upv.es). 
work has been specifically designed with manageable dimensions where any lossy liquid can be poured easily.

Furthermore, the resonant cavities proposed in most of the papers of the literature employ only symmetric modes $\mathrm{TM}_{0 \text { np }}$ or $\mathrm{TE}_{\text {0np }}[11]-[15]$. In this work, we have performed a full-wave analysis, where the whole set of modes is taken into account ( $\mathrm{TM}_{\mathrm{mn}}, \mathrm{TE}_{\mathrm{mnp}}$ and all its possible couplings and combinations, which produce hybrid modes $\mathrm{HE}_{\mathrm{mnp}}$ and $\mathrm{EH}_{\mathrm{mnp}}$ ).

A common method to analyze resonant cavities is the wellknown mode-matching because it provides very accurate results. The method consists of matching the modal fields of every region in order to accomplish the boundary conditions on the discontinuities [22]. This method is very useful, but it lacks flexibility, because one little modification of the structure implies the full reformulation of the problem. To overcome this inconvenient, generalized circuit method was developed in [23]-[24], which uses complete series of basis functions in order to generalize the analysis with basic elements characterized by admittance matrices.

In this work, the resonant structure shown in Fig 1 has been analyzed by a recently developed technique [25]-[28], which combines both mode-matching and circuit method, combining the accuracy of mode-matching with the versatility of circuit analysis.

The convergence of the solutions is discussed and a procedure to estimate the solution for an infinite number of modes is proposed. This improves the accuracy of the method and is very useful because modal methods must truncate the number of terms of the fields expansion. Furthermore, a calibration procedure is presented to minimize the error introduced by the numerical method.

In section II, the theoretical analysis is carried out with detail. Then, the study of the convergence is shown in section III. A calibration procedure is presented in section IV. Finally, and to validate the cavity cell and the analysis method, some measurements of reference liquids have been carried out in section $\mathrm{V}$.

This paper is an extension of [27], where the proposed cavity was presented for the first time. In this expanded version, a deeper explanation of theoretical analysis is shown, and the study of the convergence is carried out, which gives a complete overview of the measurement procedure. The measurement system has been calibrated to improve the accuracy. Further results include more liquids measured with several resonant frequencies.

\section{THEORETICAL MOdELING}

The geometry of the cavity analyzed in this paper is presented in Fig. 1. This structure consists of a reentrant cavity with insertion hole, where the liquid holder can be inserted easily. The main circular cavity has radius $R$ and height $H$. The reentrant metallic post has radius $r_{p}$ and height $h_{p}$. The holder has internal and external radius $r_{s}$ and $r_{t}$, respectively. The radius of the insertion hole is the same that the external radius of the holder $\left(r_{t}\right)$.

The holes in the center conductor are so small compared with the wavelength that only evanescent modes exist. Therefore, the fields are strongly attenuated inside the holes. Then, dimension $g$ must be taken large enough to ensure the complete vanishing of the fields at the end tops of the holders, so that a PEC can be considered there to simplify the analysis.

In order to develop the modal analysis, the structure has been segmented in the following independent regions:

$$
\begin{aligned}
& \text { Region 1: }\left[0<r<r_{s}\right] \cap\left[0<z_{1}<H+2 g\right] \\
& \text { Region 2: }\left[r_{s}<r<r_{t}\right] \cap\left[0<z_{2}<H+2 g\right] \\
& \text { Region 3: }\left[r_{t}<r<r_{p}\right] \cap\left[0<z_{3}<H-2 h_{p}\right] \\
& \text { Region 4: }\left[r_{p}<r<R\right] \cap\left[0<z_{4}<H\right]
\end{aligned}
$$

Every region has its own reference system of coordinates, which are related as follow:

$$
\left\{\begin{array}{c}
z_{1}=z_{2} \\
z_{3}=z_{2}-h_{p}-g \\
z_{4}=z_{3}+h_{p}
\end{array}\right.
$$

Inside every region, the full set of modes is considered, $\mathrm{TM}_{\mathrm{mnp}}$ and $\mathrm{TE}_{\mathrm{mnp}}$ modes. Thus, electric and magnetic fields inside every $i^{\text {th }}$ region can be expressed as a linear combination of TE and TM modes as follow [31].

$$
\begin{aligned}
& E_{z}^{(i)}=\sum_{m=0}^{\infty} \sum_{n=0}^{\infty}\left(\begin{array}{c}
\sin (m \varphi) \\
\cos (m \varphi)
\end{array}\right)^{T} f_{m}(r) 2 \cosh \left(\gamma_{n}^{(i)} z_{i}\right) \\
& E_{r}^{(i)}=\sum_{m=0}^{\infty} \sum_{n=0}^{\infty} \frac{-\gamma_{n}^{(i)}}{k_{c n}^{(i)}}\left(\begin{array}{c}
\sin (m \varphi) \\
\cos (m \varphi)
\end{array}\right)^{T} f_{m}^{\prime}(r)\left(-2 \sinh \left(\gamma_{n}^{(i)} z_{i}\right)\right)- \\
& \sum_{m^{\prime}=0}^{\infty} \sum_{n^{\prime}=1}^{\infty} \frac{j \omega \mu_{i}}{\left(k_{c n^{\prime}}^{(i)}\right.}\left(\begin{array}{c}
\cos \left(m^{\prime} \varphi\right) \\
-\sin \left(m^{\prime} \varphi\right)
\end{array}\right)^{T} \frac{m^{\prime}}{r} f_{m^{\prime}}(r)\left(-2 \sinh \left(\gamma_{n^{\prime}}^{(i)} z_{i}\right)\right) \\
& E_{\varphi}^{(i)}=\sum_{m=0}^{\infty} \sum_{n=0}^{\infty} \frac{-\gamma_{n}^{(i)}}{\left(k_{c n}^{(i)}\right)^{2}} \frac{m}{r}\left(\begin{array}{c}
\cos (m \varphi) \\
-\sin (m \varphi)
\end{array}\right)^{T} f_{m}(r)\left(-2 \sinh \left(\gamma_{n}^{(i)} z_{i}\right)\right) \\
& +\sum_{m^{\prime}=0}^{\infty} \sum_{n^{\prime}=1}^{\infty} \frac{j \omega \mu_{i}}{k_{c n^{\prime}}^{(i)}}\left(\begin{array}{c}
\sin \left(m^{\prime} \varphi\right) \\
\cos \left(m^{\prime} \varphi\right)
\end{array}\right)^{T} f_{m^{\prime}}^{\prime}(r)\left(-2 \sinh \left(\gamma_{n^{\prime}}^{(i)} z_{i}\right)\right)
\end{aligned}
$$$$
H_{z}^{(i)}=\sum_{m^{\prime}=0}^{\infty} \sum_{n^{\prime}=1}^{\infty}\left(\begin{array}{c}
\sin \left(m^{\prime} \varphi\right) \\
\cos \left(m^{\prime} \varphi\right)
\end{array}\right)^{T} f_{m^{\prime}}(r)\left(-2 \sinh \left(\gamma_{n^{\prime}}^{(i)} z_{i}\right)\right)
$$$$
H_{r}^{(i)}=\sum_{m=0}^{\infty} \sum_{n=0}^{\infty} \frac{j \omega \varepsilon_{i}}{\left(k_{c}^{(i)}\right)^{2}} \frac{m}{r}\left(\begin{array}{c}
\cos (m \varphi) \\
-\sin (m \varphi)
\end{array}\right)^{T} f_{m}(r) 2 \cosh \left(\gamma_{n}^{(i)} z_{i}\right)
$$$$
-\sum_{m^{\prime}=0}^{\infty} \sum_{n^{\prime}=1}^{\infty} \frac{\gamma_{n^{\prime}}^{(i)}}{k_{c n^{\prime}}^{(i)}}\left(\begin{array}{l}
\sin \left(m^{\prime} \varphi\right) \\
\cos \left(m^{\prime} \varphi\right)
\end{array}\right)^{T} f_{m^{\prime}}^{\prime}(r) 2 \cosh \left(\gamma_{n^{\prime}}^{(i)} z_{i}\right)
$$$$
H_{\varphi}^{(i)}=\sum_{m=0}^{\infty} \sum_{n=0}^{\infty} \frac{-j \omega \varepsilon_{i}}{k_{c n}^{(i)}}\left(\begin{array}{l}
\sin (m \varphi) \\
\cos (m \varphi)
\end{array}\right)^{T} f_{m}^{\prime}(r) 2 \cosh \left(\gamma_{n}^{(i)} z_{i}\right)-
$$$$
\sum_{m^{\prime}=0}^{\infty} \sum_{n^{\prime}=1}^{\infty} \frac{\gamma_{n^{\prime}}^{(i)}}{\left(k_{c n^{\prime}}^{(i)}\right)^{2}} \frac{m^{\prime}}{r}\left(\begin{array}{c}
\cos \left(m^{\prime} \varphi\right) \\
-\sin \left(m^{\prime} \varphi\right)
\end{array}\right)^{T} f_{m^{\prime}}(r) 2 \cosh \left(\gamma_{n^{\prime}}^{(i)} z_{i}\right)
$$

where the primed subscripts $m^{\prime}, n^{\prime}$ are used for TE modes and non-primed subscripts $m, n$ are associated with TM modes. Material's permittivity and permeability of every region are denoted by $\varepsilon_{i}$ and $\mu_{i}$. Propagations constants $\gamma_{n}^{(i)}$ are obtained enforcing boundary conditions of perfect electric conductor (PEC) on $z_{i}=0$ and $z_{i}=h_{i}$, where $h_{i}$ is the total height of the $i^{\text {th }}$ region $\left(h_{1}=h_{2}=H+2 g, h_{3}=H-2 h_{p}\right.$ and $\left.h_{4}=H\right)$. Thus, $\gamma_{n}^{(i)}=\frac{j n \pi}{h_{i}}$. 
Wave numbers of every region $k_{c n}^{(i)}$ are related with the propagation constant and resonant frequency as follow.

$$
\left(k_{c n}^{(i)}\right)^{2}=\left(\gamma_{n}^{(i)}\right)^{2}+\varepsilon_{i} \mu_{i}\left(2 \pi f_{r}\right)^{2}
$$

Function $f_{m}(r)$ is defined as a linear combination of Bessel functions.

$$
f_{m}(r)=A_{i} J_{m}\left(k_{c n}^{(i)} r\right)+B_{i} Y_{m}\left(k_{c n}^{(i)} r\right)
$$

where $A_{i}$ and $B_{i}$ are the amplitudes of the fields in the $i^{\text {th }}$ region, and they are vectors of dimension $2 \times 1$, whose components are the sine and cosine contributions of the electromagnetic fields. For every region, those amplitudes have a different value. In region $1, A_{1}$ is an unknown and $B_{1}=0$ because the center $r=0$ is included in the region. In regions 2 and 3, both $A_{i}$ and $B_{i}$ are unknowns. In region $4, A_{4}$ is an unknown and $B_{4}$ is determined enforcing the boundary conditions of PEC on $r=R$. Thus, $B_{4}$ has the following expressions for TM and TE modes.

$$
\begin{gathered}
B_{4}^{T M}=\frac{-J_{m}\left(k_{c n}^{(4)} R\right)}{Y_{m}\left(k_{c n}^{(4)} R\right)} \\
B_{4}^{T E}=\frac{-J_{m^{\prime}}^{\prime}\left(k_{c n^{\prime}}^{(i)} R\right)}{Y_{m^{\prime}}^{\prime}\left(k_{c n^{\prime}}^{(i)} R\right)}
\end{gathered}
$$

The function $f_{m}^{\prime}(r)$ is the first derivative of $f_{m}(r)$.

In order to match the tangential fields on every discontinuity, we have made use of circuital procedure [23]-[24], which consists of approaching the tangential fields on every $j^{\text {th }}$ interface by a complete series expansions of basis functions so that every region is characterized generically and independently of the others. The basis functions are defined as follow.

$$
\begin{gathered}
e_{z}^{(j)}=\sum_{k=0}^{\infty} \sum_{l=0}^{\infty}\left(\begin{array}{c}
\sin (k \varphi) \\
\cos (k \varphi)
\end{array}\right)^{T}\left(\begin{array}{c}
\alpha_{k l}^{s} \\
\alpha_{k l}^{c}
\end{array}\right) \cos \left(\frac{l \pi}{h_{e}} z_{e}\right) \\
e_{\varphi}^{(j)}=\sum_{k^{\prime}=0}^{\infty} \sum_{l^{\prime}=1}^{\infty}\left(\begin{array}{c}
\sin \left(k^{\prime} \varphi\right) \\
\cos \left(k^{\prime} \varphi\right)
\end{array}\right)^{T}\left(\begin{array}{c}
\beta_{k^{\prime} l^{\prime}}^{s} \\
\beta_{k^{\prime} l^{\prime}}^{c}
\end{array}\right) \sin \left(\frac{l^{\prime} \pi}{h_{e}} z_{e}\right)(13) \\
h_{z}^{(j)}=\sum_{u^{\prime}=0}^{\infty} \sum_{v^{\prime}=1}^{\infty}\left(\begin{array}{l}
\sin \left(u^{\prime} \varphi\right) \\
\cos \left(u^{\prime} \varphi\right)
\end{array}\right)^{T}\left(\begin{array}{c}
c_{u^{\prime} v^{\prime}} \\
c_{u^{\prime} v^{\prime}}^{c}
\end{array}\right) \sin \left(\frac{v^{\prime} \pi}{h_{h}} z_{h}\right) \\
h_{\varphi}^{(j)}=\sum_{u=0}^{\infty} \sum_{v=0}^{\infty}\left(\begin{array}{l}
\sin (u \varphi) \\
\cos (u \varphi)
\end{array}\right)^{T}\left(\begin{array}{c}
d_{u v}^{s} \\
d_{u v}^{c}
\end{array}\right) \cos \left(\frac{v \pi}{h_{h}} z_{h}\right)
\end{gathered}
$$

where $\alpha, \beta, c, d$ are the coefficients of the basis functions. Superscripts $s$ and $c$ refer to the sinus and cosines weights of the basis functions. Subscripts $k, l, u$ and $v$ have been associated with those of the electromagnetic fields inside of the structure as shows table I. Due to the number of modes needed in each of the regions is not the same, we have renamed the summation indexes of the fields of every $i^{\text {th }}$ region with subscripts $m_{i}$ and $n_{i}$. In the same way, the parameters $h_{e}$ and $h_{h}$ are associated with the total height of the different regions; and $z_{e}$ and $z_{h}$ are associated with the axial reference of coordinates of every region $\left(z_{i}\right)$. All these associations are shown in table I.

$$
\text { TABLE I }
$$

ASSOCIATIONS OF BASIS FUNCTIONS PARAMETERS WITH FIELDS PARAMETERS

\begin{tabular}{|c|c|c|c|c|c|c|c|c|}
\hline Interface & $k=$ & $l=$ & $u=$ & $v=$ & $h_{e}=$ & $z_{e}=$ & $h_{h}=$ & $z_{h}=$ \\
\hline$j=1$ & $m_{2}$ & $n_{2}$ & $m_{1}$ & $n_{1}$ & $h_{2}$ & $z_{2}$ & $h_{1}$ & $z_{1}$ \\
\hline$j=2$ & $m_{2}$ & $n_{2}$ & $m_{3}$ & $n_{3}$ & $h_{2}$ & $z_{2}$ & $h_{3}$ & $z_{3}$ \\
\hline$j=3$ & $m_{4}$ & $n_{4}$ & $m_{3}$ & $n_{3}$ & $h_{4}$ & $z_{4}$ & $h_{3}$ & $z_{3}$ \\
\hline
\end{tabular}

Now, instead of matching directly the tangential fields on every discontinuity such as in classical mode-matching method, the tangential fields of each of the regions are approached on every interface by the basis function defined above. This procedure consists of generalize the classical mode-matching method using generic basis functions, since the basis functions are unalterable, while the fields change for every little modification of the structure. Then, we must enforce the following equalities on the discontinuities or interfaces.

Interface $j=1, r=r_{s}$

$$
\begin{aligned}
& \left.E_{z}^{(1)}\right|_{r=r_{s}}=e_{z}^{(1)} \text { and }\left.E_{z}^{(2)}\right|_{r=r_{s}}=e_{z}^{(1)} \\
& \left.E_{\varphi}^{(1)}\right|_{r=r_{s}}=e_{\varphi}^{(1)} \text { and }\left.E_{\varphi}^{(2)}\right|_{r=r_{s}}=e_{\varphi}^{(1)} \\
& \left.H_{z}^{(1)}\right|_{r=r_{s}}=h_{z}^{(1)} \text { and }\left.H_{z}^{(2)}\right|_{r=r_{s}}=h_{z}^{(1)} \\
& \left.H_{\varphi}^{(1)}\right|_{r=r_{s}}=h_{\varphi}^{(1)} \text { and }\left.H_{\varphi}^{(2)}\right|_{r=r_{s}}=h_{\varphi}^{(1)}
\end{aligned}
$$

Interface $j=2, r=r_{t}$

$$
\begin{aligned}
& \left.E_{z}^{(2)}\right|_{r=r_{t}}=e_{z}^{(2)} \text { and }\left.E_{z}^{(3)}\right|_{r=r_{t}}=e_{z}^{(2)} \\
& \left.E_{\varphi}^{(2)}\right|_{r=r_{t}}=e_{\varphi}^{(2)} \text { and }\left.E_{\varphi}^{(3)}\right|_{r=r_{t}}=e_{\varphi}^{(2)} \\
& \left.H_{z}^{(2)}\right|_{r=r_{t}}=h_{z}^{(2)} \text { and }\left.H_{z}^{(3)}\right|_{r=r_{t}}=h_{z}^{(2)} \\
& \left.H_{\varphi}^{(2)}\right|_{r=r_{t}}=h_{\varphi}^{(2)} \text { and }\left.H_{\varphi}^{(3)}\right|_{r=r_{t}}=h_{\varphi}^{(2)}
\end{aligned}
$$

Interface $j=3, r=r_{p}$

$$
\begin{aligned}
& \left.E_{z}^{(3)}\right|_{r=r_{p}}=e_{z}^{(3)} \text { and }\left.E_{z}^{(4)}\right|_{r=r_{p}}=e_{z}^{(3)} \\
& \left.E_{\varphi}^{(3)}\right|_{r=r_{p}}=e_{\varphi}^{(3)} \text { and }\left.E_{\varphi}^{(4)}\right|_{r=r_{p}}=e_{\varphi}^{(3)} \\
& \left.H_{z}^{(3)}\right|_{r=r_{p}}=h_{z}^{(3)} \text { and }\left.H_{z}^{(4)}\right|_{r=r_{p}}=h_{z}^{(3)} \\
& \left.H_{\varphi}^{(3)}\right|_{r=r_{p}}=h_{\varphi}^{(3)} \text { and }\left.H_{\varphi}^{(4)}\right|_{r=r_{p}}=h_{\varphi}^{(3)}
\end{aligned}
$$

Applying the orthogonal properties of the basis functions, the coefficients $\alpha, \beta, c, d$ can be obtained in two different ways (once per each side of the interface).

Interface $j=1, r=r_{s}$

$$
\begin{aligned}
& \alpha_{1}=\left\langle\left. E_{z}^{(1)}\right|_{r=r_{s}}{ }^{\prime} \tilde{e}_{z}^{(1)}\right\rangle \text { and } \alpha_{1}=\left\langle\left. E_{z}^{(2)}\right|_{r=r_{s}}{ }^{,} \tilde{e}_{z}^{(1)}\right\rangle \\
& \beta_{1}=\left\langle\left. E_{\varphi}^{(1)}\right|_{r=r_{s}}, \tilde{e}_{\varphi}^{(1)}\right\rangle \text { and } \beta_{1}=\left\langle\left. E_{\varphi}^{(2)}\right|_{r=r_{s}}, \tilde{e}_{\varphi}^{(1)}\right\rangle \\
& c_{1}=\left\langle\left. H_{z}^{(1)}\right|_{r=r_{s}} \tilde{h}_{z}^{(1)}\right\rangle \text { and } c_{1}=\left\langle\left. H_{z}^{(2)}\right|_{r=r_{s}}{ }^{\prime} \tilde{h}_{z}^{(1)}\right\rangle \\
& d_{1}=\left\langle\left. H_{\varphi}^{(1)}\right|_{r=r_{s}}{ }^{\prime} \tilde{h}_{\varphi}^{(1)}\right\rangle \text { and } d_{1}=\left\langle\left. H_{\varphi}^{(2)}\right|_{r=r_{s}}{ }^{\prime} \tilde{h}_{\varphi}^{(1)}\right\rangle
\end{aligned}
$$

Interface $j=2, r=r_{t}$

$$
\begin{aligned}
& \alpha_{2}=\left\langle\left. E_{z}^{(2)}\right|_{r=r_{t}} \tilde{e}_{z}^{(2)}\right\rangle \text { and } \alpha_{2}=\left\langle\left. E_{z}^{(3)}\right|_{r=r_{t}{ }^{\prime}} \tilde{e}_{z}^{(2)}\right\rangle \\
& \beta_{2}=\left\langle\left. E_{\varphi}^{(2)}\right|_{r=r_{t}}{ }^{\prime} \tilde{e}_{\varphi}^{(2)}\right\rangle \text { and } \beta_{2}=\left\langle\left. E_{\varphi}^{(3)}\right|_{r=r_{t}}{ }^{\prime} \tilde{e}_{\varphi}^{(2)}\right\rangle \\
& c_{2}=\left\langle\left. H_{z}^{(2)}\right|_{r=r_{t}}{ }^{\prime} \tilde{h}_{z}^{(2)}\right\rangle \text { and } c_{2}=\left\langle\left. H_{z}^{(3)}\right|_{r=r_{t}}{ }^{\prime} \tilde{h}_{z}^{(2)}\right\rangle \\
& d_{2}=\left\langle\left. H_{\varphi}^{(2)}\right|_{r=r_{t}}{ }^{\prime} \tilde{h}_{\varphi}^{(2)}\right\rangle \text { and } d_{2}=\left\langle\left. H_{\varphi}^{(3)}\right|_{r=r_{t}}{ }^{\prime} \tilde{h}_{\varphi}^{(2)}\right\rangle
\end{aligned}
$$

Interface $j=3, r=r_{p}$

$$
\begin{aligned}
& \alpha_{3}=\left\langle\left. E_{z}^{(3)}\right|_{r=r_{p}}, \tilde{e}_{z}^{(3)}\right\rangle \text { and } \alpha_{3}=\left\langle\left. E_{z}^{(4)}\right|_{r=r_{p}}, \tilde{e}_{z}^{(3)}\right\rangle \\
& \beta_{3}=\left\langle\left. E_{\varphi}^{(3)}\right|_{r=r_{p}}, \tilde{e}_{\varphi}^{(3)}\right\rangle \text { and } \beta_{3}=\left\langle\left. E_{\varphi}^{(4)}\right|_{r=r_{p}}, \tilde{e}_{\varphi}^{(3)}\right\rangle \\
& c_{3}=\left\langle\left. H_{z}^{(3)}\right|_{r=r_{p}}, \tilde{h}_{z}^{(3)}\right\rangle \text { and } c_{3}=\left\langle\left. H_{z}^{(4)}\right|_{r=r_{p}}, \tilde{h}_{z}^{(3)}\right\rangle \\
& d_{3}=\left\langle\left. H_{\varphi}^{(3)}\right|_{r=r_{p}}, \tilde{h}_{\varphi}^{(3)}\right\rangle \text { and } d_{3}=\left\langle\left. H_{\varphi}^{(4)}\right|_{r=r_{p}}, \tilde{h}_{\varphi}^{(3)}\right\rangle
\end{aligned}
$$

where $\langle\cdot, \cdot\rangle$ denotes the inner product operation, and $\tilde{e}$ and $\tilde{h}$ denotes the expressions of the basis functions (12)-(15) without 
the coefficients $\alpha, \beta, c, d$.

Then, from the previous equations, a system of matrix equations can be formulated, where the unknowns are amplitudes $\left(A_{i}\right.$ and $\left.B_{i}\right)$ of the fields $\left(E^{(i)}, H^{(i)}\right)$ in every region. Since there are amplitudes for TM and TE modes, we are going to define the following vectors of amplitudes in every region of the structure in order to simplify the notation:

$$
\begin{aligned}
& a_{1}=\left[A_{1}^{T M}, A_{1}^{T E}\right] \\
a_{2}= & {\left[A_{2}^{T M}, A_{2}^{T E}, B_{2}^{T M}, B_{2}^{T E}\right] } \\
a_{3}= & {\left[A_{3}^{T M}, A_{3}^{T E}, B_{3}^{T M}, B_{3}^{T E}\right] } \\
& a_{4}=\left[A_{4}^{T M}, A_{4}^{T E}\right]
\end{aligned}
$$

The resulting system of matrix equations is shown in (44), where one can notice that there are 12 equations for 12 unknown amplitudes.

$$
\left\{\begin{array}{l}
F_{1} \cdot a_{1}=F_{2} \cdot a_{2} \\
F_{3} \cdot a_{1}=F_{4} \cdot a_{2} \\
G_{1} \cdot a_{1}=G_{2} \cdot a_{2} \\
G_{3} \cdot a_{1}=G_{4} \cdot a_{2} \\
Q_{1} \cdot a_{2}=Q_{2} \cdot a_{3} \\
Q_{3} \cdot a_{2}=Q_{4} \cdot a_{3} \\
R_{1} \cdot a_{2}=R_{2} \cdot a_{3} \\
R_{3} \cdot a_{2}=R_{4} \cdot a_{3} \\
S_{1} \cdot a_{3}=S_{2} \cdot a_{4} \\
S_{3} \cdot a_{3}=S_{4} \cdot a_{4} \\
T_{1} \cdot a_{3}=T_{2} \cdot a_{4} \\
T_{3} \cdot a_{3}=T_{4} \cdot a_{4}
\end{array}\right.
$$

The expressions of matrices $F, G, Q, R, S$ and $T$ are obtained solving the inner products shown in (28) to (39).

The previous system of equation can be rewritten in a more compact form using matrix notation.

$$
X \cdot \vec{a}=0
$$

where $\vec{a}$ is a column vector with the whole set of unknown amplitudes of the fields inside the analyzed structure.

Last equation can be associated with an eigenvalue problem, whose non-trivial solutions are given by the roots of the absolute value of the determinant of $X$.

$$
|\operatorname{det}[X]|=0
$$

In this formula, $X$ is a function of geometric dimensions of the cavity, resonant frequency and material properties of the different regions (permittivity and permeability). In resonant problems, the direct or forward solution consists of obtaining the resonant frequencies of the structure from the known material parameters and geometric dimensions, whereas the inverse or backward solution consists of obtaining any other parameter (permittivity, permeability or geometric dimensions) from the known resonant frequency [22]. This fact is very important, since modal methods provides this possibility unlike the classical FEM or FDTD methods employed by commercial software. More detailed explanation about eigenvalue problem of mode-matching is provided in [29]-[ 30].

\section{STUdY OF THE CONVERGENCE}

In the resolution of (45), the infinite series of fields expansions must be truncated to solve viably the system of equations. Then, in equations (2)-(7), summation of angular variable $\varphi$, whose subscript is $m_{i}$, is truncated to $M_{i}$ terms, and summation of axial variable $z_{i}$, whose subscript is $n_{i}$, is truncated to $N_{i}$ terms. Then, the total number of modes considered in the $i^{\text {th }}$ region is $M_{i} \times N_{i}$.

The truncation of the series has an important influence on the convergence behavior. For this reason, it is important to study carefully the convergence of the proposed method with the analyzed cavity. In fact, two important aspects have been considered in this paper. First, the relation between the modes of the different regions must be established properly in order to ensure the fastest and most accurate convergence of the method. Secondly, we have provided a fitting procedure with which the solution of the problem calculated with infinite number of modes can be achieved. This fact is very important because of the impossibility of applying the method with infinite number of modes, not even with a very high number of modes due to numerical problems produced by solving (46).

The modal analysis performed in this section is a variant performance of the classical mode-matching method, but at the end, the procedure is analogous. For this reason, the proposed method is under the influence of the well-known relative convergence of the mode-matching, which establishes that the solution converges to a different set of answers for every different choice of modes in each of the regions [32].

The establishment of the relation between the modes of different regions has been studied by many authors for many years [32]-[35]. All of them agree with the principle that the convergence is ensured when the wavelength spatial resolution is the same in both sides of the discontinuity, but it is difficult to find a useful expression to apply it in practice. Especially interesting is the contribution of [35], which gives an optimal and practical expression using only the dimensions of the waveguide $\left(d_{i}\right)$ and the number of modes $\left(P_{i}\right)$ in the $i^{\text {th }}$ region.

$$
\frac{P_{i}}{d_{i}}=\frac{P_{i+1}}{d_{i+1}}
$$

Due to the full-wave analysis that we have performed, two set of indexes are employed (subscripts $m$ and $n$ of equations (2)-(7)), one for angular dimension ( $\varphi \rightarrow m$ ) and the other one for axial dimension $(z \rightarrow n)$. Because the analyzed structure is axially symmetric, the number of variations in $\varphi$ dimension $\left(M_{i}\right)$ must be the same in all the regions, whereas the number of variations in $z$ dimension $\left(N_{i}\right)$ is directly related with the total heights of the regions:

$$
\left\{\begin{array}{c}
\frac{M_{i}}{2 \pi}=\frac{M_{i+1}}{2 \pi} \rightarrow M_{i}=M_{i+1} \\
\frac{N_{i}}{h_{i}}=\frac{N_{i+1}}{h_{i+1}} \rightarrow N_{i+1}=\operatorname{ceil}\left(\frac{h_{i+1}}{h_{i}} N_{i}\right)
\end{array}\right.
$$

Then, the number of modes of every region can be obtained recursively applying (48). Due to the number of modes must be integer, ceiling function $(c e i l(x))$ is used, which rounds to the nearest integer greater than or equal to $x$.

It is important to remark that relation (47) is only satisfied when the number of modes is large enough in both sides of the junction. Then, that expression gives the optimal convergence relation between the modes of the different regions. For the particular analyzed cavity, the minimum number of modes considered to guarantee an acceptably close value of the convergent solution must be $M_{1}=M_{2}=M_{3}=M_{4}=4$ and $N_{1}=N_{2}=10, N_{3}=4, N_{4}=10$. 
With the optimal relation of the number of modes obtained above, the problem of the relative convergence is solved. Then, from the rigorous point of view, the most accurate solution would be provided considering infinite number of modes or terms in the summation of the fields expansions. This case cannot be treated computationally by the direct way (solving (46)). Nevertheless, we can study the behavior of the solutions provided by the method depending on the number of modes considered, with the purpose of determining the asymptotic behavior of the solutions.

To this aim, we have considered the empty cavity, without holder and material, with the purpose of simplifying the analysis, since the behavior of the method for the loaded cavity is completely analogous. Nominal geometric dimensions of the cavity are the following: $H=119.7 \mathrm{~mm}, R=60 \mathrm{~mm}, h_{p}=35.65 \mathrm{~mm}$, $r_{p}=24 \mathrm{~mm}, r_{t}=6 \mathrm{~mm}$.

In Fig. 2, the calculated resonant frequencies as a function of the modes considered in fields expansion is shown. The number of terms of angular variation has been fixed to $M_{i}=4$ in all the regions. The number of modes shown in the graph are the axial number of terms of the region $3\left(N_{3}\right)$, the number of modes of the other regions can be obtained with (48). Only the first resonant frequencies of the empty cavity are shown, but the following ones have the same tendency.
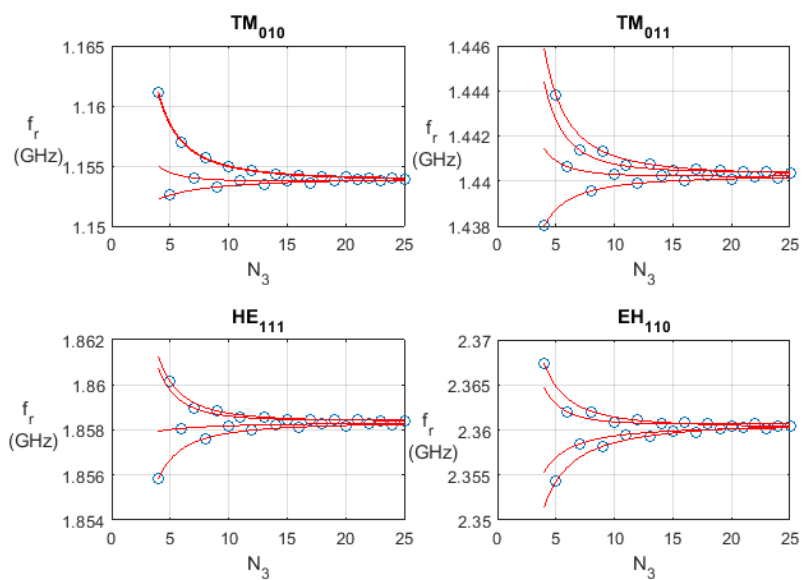

Fig. 2. Behavior of the computed resonant frequencies as a function of the number of modes considered in simulation, and asymptotic fitting curves.

The correct convergence of the method is noticed because the more modes in the simulation, the less variance of the solutions.

Taking the data with proper periodicity, very clear convergent curves are obtained, which can be fitted with the following nonlinear regression function:

$$
y=\frac{a_{1}}{x^{2}}+\frac{a_{2}}{x}+a_{3}
$$

where $a_{1}, a_{2}$ and $a_{3}$ are the fitting coefficients, $y$ is the value of the resonant frequency and $x$ is the number of modes considered in simulations. Therefore, the asymptotic value of the computed resonant frequency considering infinite number of modes $(x \rightarrow$ $\infty$ ) is given by the parameter $a_{3}$ of every curve.

Periodicity of the data is given by the optimal relation of the modes. In this particular case, the optimal relation in region 3 can be expressed as follow:

$$
N_{3}=\frac{h_{3}}{h_{4}} N_{4}=\frac{H-2 h_{p}}{H} N_{4} \cong 0.4 N_{4} \rightarrow \frac{N_{3}}{N_{4}}=\frac{4}{10}
$$

This expression means that, taking the minimum value in region $3\left(N_{3}=4\right)$, for every 4 modes on region 3 , there must be 10 in region 4. All the following optimal relations that ensure the convergence of the method with the same asymptotic behavior must be multiples of (50) (i.e. $8 / 20,12 / 30,16 / 40 \ldots$ ), because the same ratio and the same contribution of the cutoff high order modes must be accomplished. Then, in this particular case, the period of the data is 4 , because simulations are performed as a function of $N_{3}$. If the simulation would be performed as a function of $N_{4}$, period would be 10 , which means that ten different asymptotic curves would appear.

Thus, due to the periodicity of the data, there are four different curves for each resonant frequency, and consequently, four different asymptotic values. Then, we have calculated the arithmetic mean and the standard deviation of those values to determine the final asymptotic value and its uncertainty, respectively. The standard deviation of the asymptotic approximation is considered an independent source of uncertainty in the measurement $(u(x))$.

The study of the convergence carried out in this section has been analyzed with forward formulation of the resonant problem. For the backward formulation, the convergent behavior of the solution is analogous.

\section{CALIBRATION PROCEDURE}

The proposed theoretical method considers ideal conditions of the problem, but it does not take into account the imperfections of the geometry produced in any manufacture process, such as rugosity of the walls, lack of concentricity of all the parts of the cavity, imperfect cylinders, dilatation and contraction of the metallic walls etc. Besides, the method has the assumable error uncertainty of any numerical technique. Therefore, the calibration of the measurement system is recommended to avoid accumulative errors in high precision measurements.

The calibration procedure consists of estimating all the dimensions of the cavity indirectly from the measured resonant frequencies of the empty resonator. For this purpose, a system of nonlinear equations must be formulated in the following form:

$$
\left\{\begin{array}{c}
y=F_{1}\left(f_{1}, r_{c}, h_{c}, h_{p}, r_{p}, r_{t}\right) \\
y=F_{2}\left(f_{2}, r_{c}, h_{c}, h_{p}, r_{p}, r_{t}\right) \\
y=F_{N-1}\left(f_{N-1}, r_{c}, h_{c}, h_{p}, r_{p}, r_{t}\right) \\
y=F_{N}\left(f_{N}, r_{c}, h_{c}, h_{p}, r_{p}, r_{t}\right)
\end{array}\right.
$$

where $F_{i}\left(f_{i}, r_{c}, h_{c}, h_{p}, r_{p}, r_{t}\right)$ is a function depending on the measured resonant frequency $\left(f_{i}\right)$ and dimensions of the cavity, and its value is given by (46). There are $N$ equations, where $N$ must be greater or equal to the number of dimensions to determine. In this case, we have taken 8 equations, one per measured resonant frequency with the empty cavity.

The optimization function to achieve the dimensions of the cavity indirectly from the measured resonant frequencies is described as follows: 


$$
\sum_{i=1}^{N} F_{i}=0
$$

The variables to determine with any optimization procedure are the dimensions of the cavity, whose values can be understood as the effective dimensions of the cavity, with which the measured resonant frequencies of the empty cavity are adjusted.

Comparison of the nominal and effective geometric dimensions is shown in table II. Effective dimensions have been obtained with a generic simplex search optimization method described in [36]. The uncertainty of the nominal values is given by the digital caliper, and the uncertainty of the effective values is given by the standard deviation of the solutions provided in simulations with different number of modes. Nominal and effective values are very close as expected. The little differences between them $(\mu m)$ adjust the resonant frequency of all the modes of the empty cavity as shown in table III and IV.

TABLE II

NOMINAL AND EFFECTIVE GEOMETRIC DIMENSIONS OF THE CAVITY

\begin{tabular}{|c|c|c|c|c|}
\hline Dimension & $\begin{array}{c}\text { Nominal } \\
\text { Value }(\mathrm{mm})\end{array}$ & $\begin{array}{c}u(x) \\
(\mu \mathrm{m})\end{array}$ & $\begin{array}{c}\text { Effective } \\
\text { Value }(\mathrm{mm})\end{array}$ & $\begin{array}{c}u(x) \\
(\mu \mathrm{m})\end{array}$ \\
\hline$H$ & 119.7 & \pm 50 & 119.7324 & \pm 6 \\
\hline$R$ & 60 & \pm 50 & 60.0206 & \pm 7 \\
\hline$h_{p}$ & 35.65 & \pm 50 & 35.6542 & \pm 3 \\
\hline$r_{p}$ & 24 & \pm 50 & 24.0149 & \pm 5 \\
\hline$r_{t}$ & 6 & \pm 50 & 6.0587 & \pm 3 \\
\hline
\end{tabular}

In table III, the computed resonant frequencies of the first 8 modes considering nominal and effective dimensions are shown, as well as the uncertainty provided by the asymptotic estimation of the solutions. The uncertainty of the computed resonant frequencies is reduced with the effective dimensions, as expected. In table IV, the first 8 measured resonant frequencies are shown, as well as the absolute error with simulations of nominal and effective dimensions. Results show that the effective dimensions minimize the error between simulation and measurement.

TABLE III

COMPUTED RESONANT FREQUENCIES WITH NOMINAL AND EFFECTIVE DIMENSIONS OF THE EMPTY CAVITY

\begin{tabular}{|l|c|c|c|c|}
\hline Mode & $\begin{array}{c}f_{r}(\mathrm{GHz})- \\
\text { Nominal } \\
\text { Geometric } \\
\text { Dimensions }\end{array}$ & $\begin{array}{c}u\left(f_{r}\right) \\
(\mathrm{MHz})\end{array}$ & $\begin{array}{c}f_{r}(\mathrm{GHz})- \\
\text { Effective } \\
\text { Geometric } \\
\text { Dimensions. }\end{array}$ & $\begin{array}{c}u\left(f_{r}\right) \\
(\mathrm{MHz})\end{array}$ \\
\hline $\mathrm{TM}_{010}$ & 1.153863 & \pm 0.5 & 1.154109 & \pm 0.1 \\
\hline $\mathrm{TM}_{011}$ & 1.440280 & \pm 0.3 & 1.440509 & \pm 0.09 \\
\hline $\mathrm{HE}_{111}$ & 1.858386 & \pm 0.3 & 1.858487 & \pm 0.03 \\
\hline $\mathrm{EH}_{110}$ & 2.360553 & \pm 0.6 & 2.360790 & \pm 0.05 \\
\hline $\mathrm{HE}_{211}$ & 2.678205 & \pm 0.4 & 2.678185 & \pm 0.2 \\
\hline $\mathrm{TM}_{012}$ & 2.858571 & \pm 0.5 & 2.858663 & \pm 0.2 \\
\hline $\mathrm{EH}_{212}$ & 3.295091 & \pm 0.4 & 3.294995 & \pm 0.1 \\
\hline $\mathrm{EH}_{111}$ & 3.410385 & \pm 0.5 & 3.410348 & \pm 0.2 \\
\hline
\end{tabular}

TABLE IV

ERROR OF THE CALCULATED RESONANT FREQUENCIES IN REFERENCE WITH THE MEASUREMENTS

\begin{tabular}{|c|c|c|c|}
\hline Mode & $\begin{array}{c}f_{r}(\mathrm{GHz})- \\
\text { Measured }\end{array}$ & $\begin{array}{c}\Delta f_{r}(\mathrm{MHz})- \\
\text { Nominal } \\
\text { Geometric } \\
\text { Dimensions }\end{array}$ & $\begin{array}{c}\Delta f_{r}(\mathrm{MHz})- \\
\text { Effective } \\
\text { Geometric } \\
\text { Dimensions. }\end{array}$ \\
\hline $\mathrm{TM}_{010}$ & 1.154188 & 0.326 & 0.079 \\
\hline $\mathrm{TM}_{011}$ & 1.440528 & 0.248 & 0.019 \\
\hline $\mathrm{HE}_{111}$ & 1.858478 & 0.092 & 0.009 \\
\hline $\mathrm{EH}_{110}$ & 2.361368 & 0.815 & 0.578 \\
\hline $\mathrm{HE}_{211}$ & 2.678175 & 0.031 & 0.010 \\
\hline $\mathrm{TM}_{012}$ & 2.858647 & 0.075 & 0.016 \\
\hline $\mathrm{EH}_{212}$ & 3.294741 & 0.350 & 0.254 \\
\hline $\mathrm{EH}_{111}$ & 3.409771 & 0.614 & 0.577 \\
\hline
\end{tabular}

The identification of the modes has been carried out by plotting the field distribution inside the cavity for each resonant frequency. In Fig. 3 to 5, the field distribution of some modes inside of the empty cavity is shown. The modes can be identified as transversal electric ( $\mathrm{TE}_{\mathrm{mn}}$ ), which lack of axial electric contribution $\left(E_{\mathrm{z}}\right)$; transversal magnetic $\left(\mathrm{TM}_{\mathrm{mnp}}\right)$, which lack of axial magnetic contribution $\left(H_{z}\right)$; and hybrids $\left(\right.$ HEM $\left._{\mathrm{mnp}}\right)$, which have both axial electric and magnetic contribution. In hybrids modes, when $E_{z}$ predominate over $H_{z}$, the modes are call $\mathrm{EH}_{\text {mnp }}$. Otherwise, when $H_{z}$ predominate over $E_{z}$, the modes are call $\mathrm{HE}_{\mathrm{mnp}}$. Subscripts $m, n$ and $p$ show, respectively, the number of field variations in $\varphi, r$ and $z$ dimensions [31].

In Fig 3.a, at $1.44 \mathrm{GHz}$, the representation of the components of the electric field on a transversal plane shows that there are not variations on angular dimension $(m=0)$ and there is one variation in radial dimension ( $(n=1)$. In Fig 3.b, $H_{\varphi}$ shows there is one variation in axial dimension $(p=1)$. Due to this first mode has a negligible axial component of magnetic field, we can identify this frequency as a transversal magnetic $\mathrm{TM}_{011}$ mode. In Fig 4.a, at $2.678 \mathrm{GHz}$, the representation of the components of the electric field on a transversal plane shows that there are two variations on angular dimension $(m=2)$ and there is one variation in radial dimension ( $n=1)$. In Fig 4.b, the components of the magnetic field show that there is one variation in axial dimension $(p=1)$. Due to that mode has both axial component of electric and magnetic field, we can identify this frequency as a hybrid mode, and because $H_{z}$ has a bigger contribution over the other components of magnetic field than $E_{z}$ over the other components of electric field, following notation of [31], we have named this mode $\mathrm{HE}_{211}$. In Fig 5.a, at $3.295 \mathrm{GHz}$, the representation of the components of the electric field on a transversal plane shows that there are two variations on angular dimension $(m=2)$ and there is one variation in radial dimension $(n=1)$. In Fig 5.b, the components of the magnetic field show that there are two variations in axial dimension $(p=2)$. Due to that mode has both axial component of electric and magnetic field, we can identify this frequency as a hybrid mode, and because $E_{z}$ has a bigger contribution over the other components of electric field than $\mathrm{H}_{\mathrm{z}}$ over the other components of magnetic field, following notation of [31], we have named this mode $\mathrm{EH}_{212}$. 

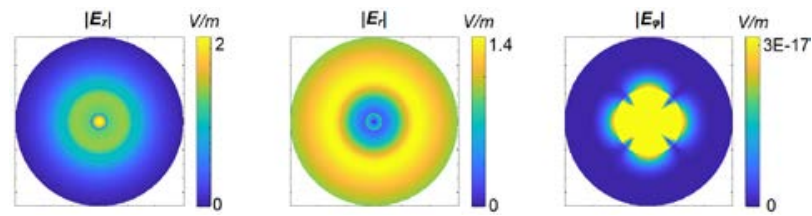

(a)
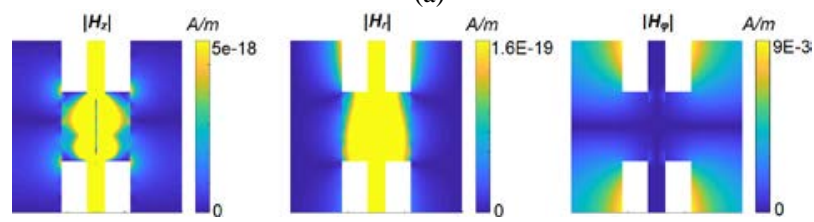

(b)

Fig. 3. Field distribution at $f_{r}=1.44 \mathrm{GHz}$, mode $\mathrm{TM}_{011}$. (a) Electric field components on $z_{4}=1.25 h_{p}$ (b) Magnetic field components on $\varphi=\pi / 7$
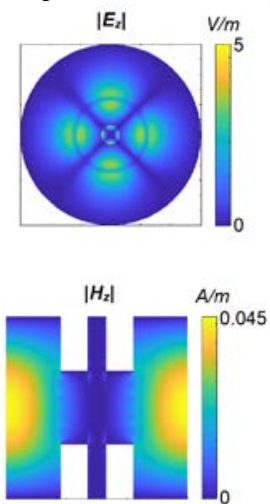

Fig. 4. Field distribution at $f_{r}=2.678 \mathrm{GHz}$, mode $\mathrm{HE}_{211}$. (a) Electric field components on $z_{4}=1.25 h_{p}$ (b) Magnetic field components on $\varphi=\pi / 7$
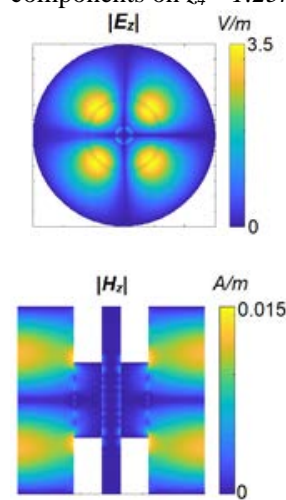

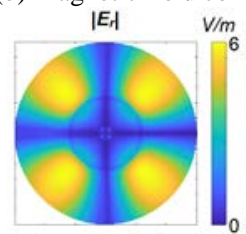

(a)

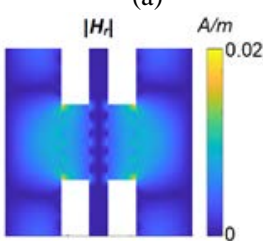

(b)
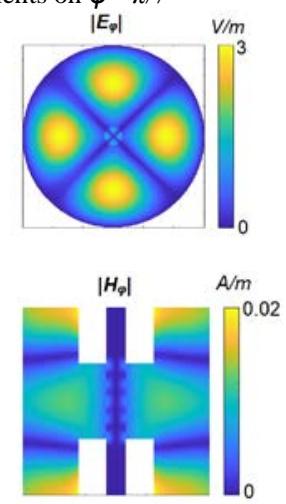

Fig. 5. Field distribution at $f_{r}=3.295 \mathrm{GHz}$, mode $\mathrm{EH}_{212}$. (a) Electric field components on $z_{4}=1.25 h_{p}$ (b) Magnetic field components on $\varphi=\pi / 7$

\section{EXPERIMENTAL RESULTS AND DISCUSSION}

To measure the complex permittivity of a liquid, it is poured in a holder, whose external radius coincides with the radius of the insertion holes, $r_{t}$, and the internal radius is $r_{s}=4.9 \pm 0.05 \mathrm{~mm}$. Quartz has been chosen as the material of the holder because its low losses improve the quality factor [12].

The election of the most appropriate modes to measure high loss liquids is crucial in dielectrometry application, since the whole set of modes are not suitable to measure the complex permittivity of those liquids.

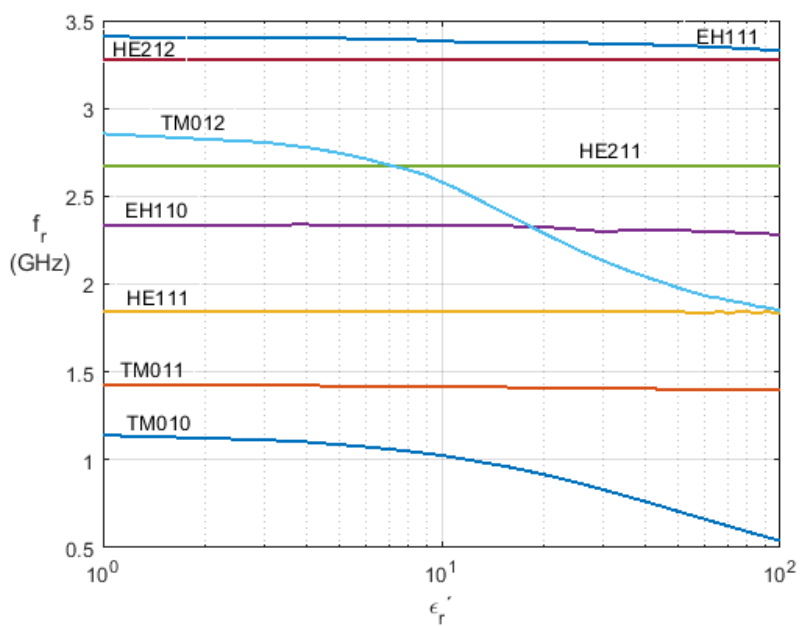

(a)

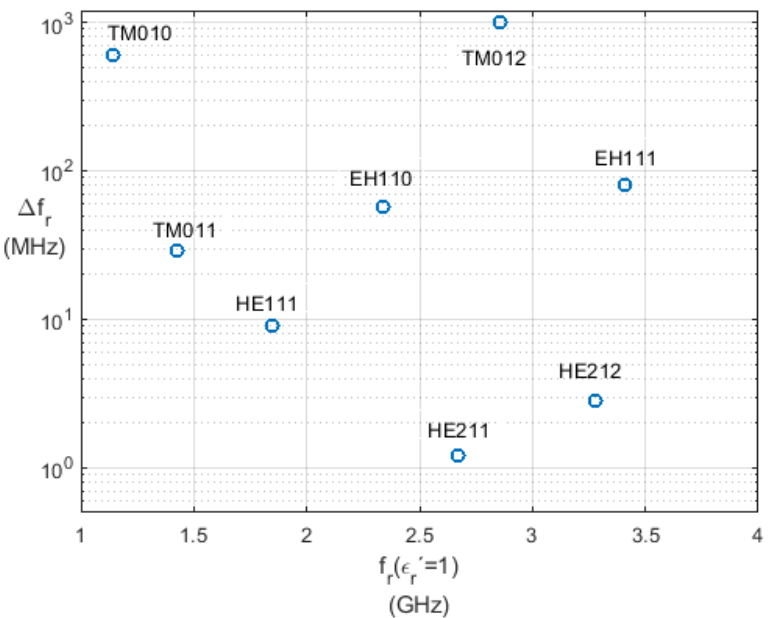

(b)

Fig. 6. (a) Variation of the resonant frequency as a function of the dielectric constant of the sample. (b) Absolute difference of the resonant frequency with $\varepsilon_{r}^{\prime}=1$ and $\varepsilon_{r}^{\prime}=50$.

In Fig. 6, the sensitivity of the resonant frequency of every mode is shown as a function of the dielectric constant $\left(\varepsilon_{r}^{\prime}\right)$ of the liquid. The more variation in the resonant frequency, the better results provides the mode on the estimation of the dielectric constant, since a little change on $\varepsilon_{r}^{\prime}$ is noticed in the resonant frequency, being those modes more sensitive than others. Modes $\mathrm{TM}_{0 \mathrm{np}}$ and $\mathrm{EH}_{\mathrm{mnp}}$ are the most sensitive in this sense, while $\mathrm{HE}_{\mathrm{mnp}}$ modes do not have enough sensitivity to measure accurately the dielectric constant of the liquids.

In addition, the quality factor must be large enough to be measured. In Fig. 7, the variation of the quality factor $\left(Q_{d}\right)$ associated with the dielectric losses $\left(\varepsilon_{r}^{\prime \prime}\right)$ is shown for some fixed values of the dielectric constant: $\varepsilon_{r}^{\prime}=[2,20,50]$. Modes $\mathrm{TM}_{0 \mathrm{np}}$ and $\mathrm{EH}_{\mathrm{mnp}}$ have generally a steep slope, while $\mathrm{HE}_{\mathrm{mnp}}$ are more constant. For high losses, modes $\mathrm{TM}_{010}$ and $\mathrm{TM}_{012}$ have too low $Q_{d}$ to be measured accurately. As Fig. 7.a to 7.c show, the behavior of the quality factor of every mode is similar in the whole range of $\varepsilon_{r}^{\prime}$, hence the discussion performed for these particular cases $\left(\varepsilon_{r}^{\prime}=[2,20,50]\right)$ can be extrapolated for other values of $\varepsilon_{r}^{\prime}$. 


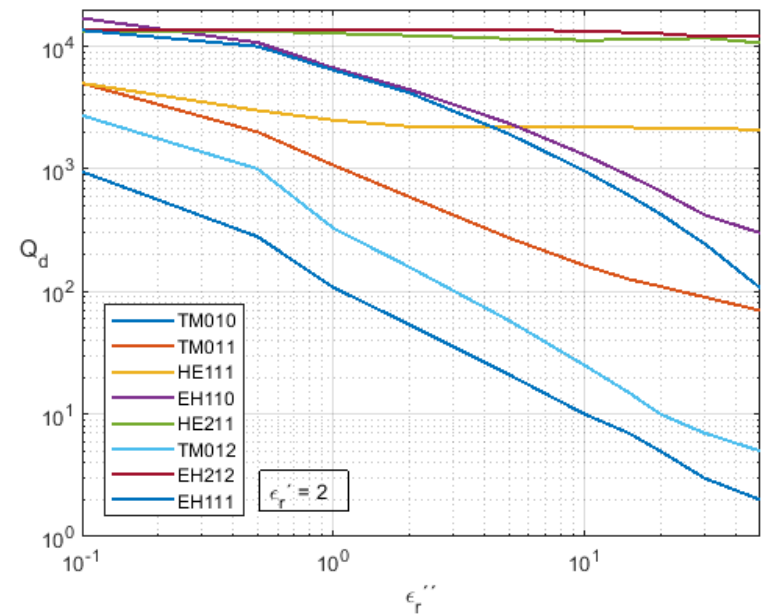

(a)

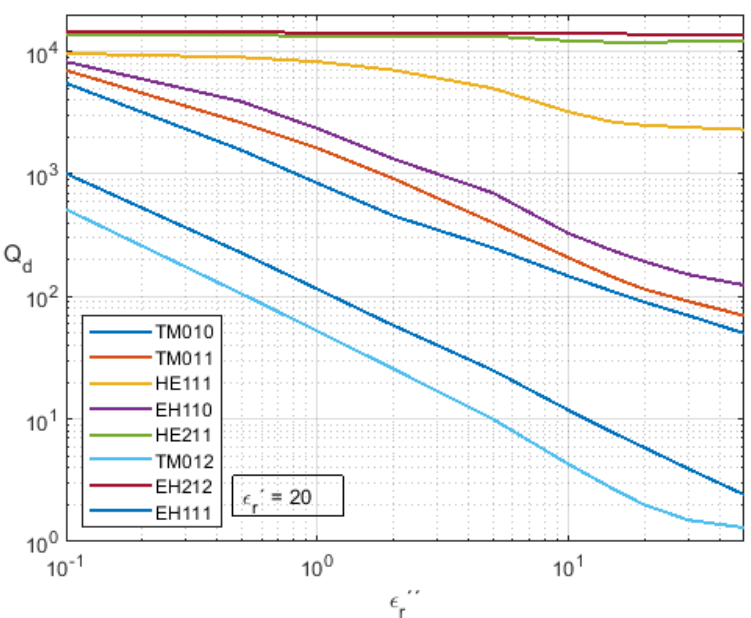

(b)

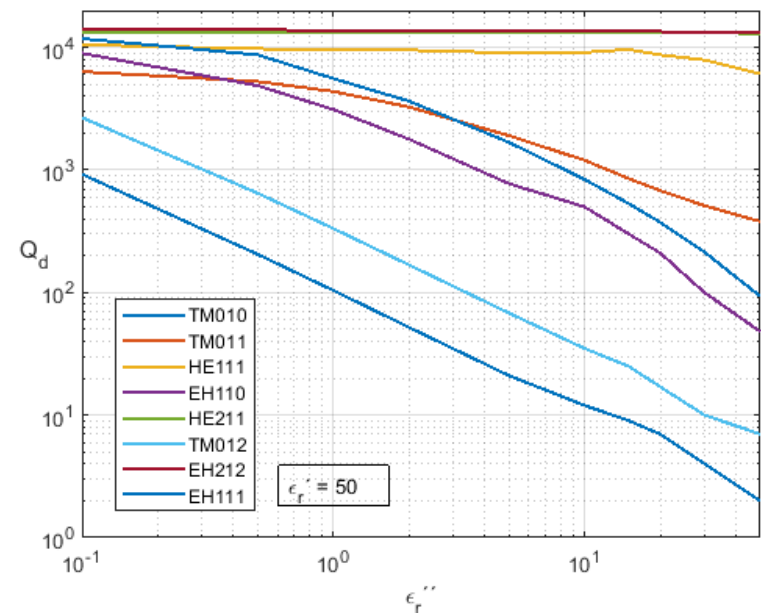

(c)

Fig. 7. Variation of the quality factor associated with the dielectric losses $\left(Q_{d}\right)$, versus with the loss factor of the sample with: (a) $\varepsilon_{r}^{\prime}=2$, (b) $\varepsilon_{r}^{\prime}=20$, and (c) $\varepsilon_{r}^{\prime}=50$.

On the basis of the previous discussion, $\mathrm{TM}_{011}$ and the $\mathrm{EH}_{110}$ modes are the selected modes to measure the complex permittivity of some reference liquids. The modes $\mathrm{HE}_{\mathrm{mn}}$ have been discarded due to the poor sensitivity of the resonant frequency shown in Fig. 6. On the other hand, modes $\mathrm{TM}_{010}$ and
$\mathrm{TM}_{012}$ have been discarded due to the low quality factor provided with high losses. $\mathrm{EH}_{111}$ is a good mode to measure the complex permittivity, but the coupling of the employed probe is not strong enough to excite the mode properly when a liquid is inserted in the cavity.

The resonant frequency and quality factor have been measured with a vector network analyzer (VNA). The unloaded measurements of the resonator $\left(f_{u}\right.$ and $\left.Q_{u}\right)$ have been obtained applying the procedure described in [37]-[38], where the effect of the coupling is removed. The unloaded quality factor $\left(Q_{u}\right)$ can be expressed as follows:

$$
\frac{1}{Q_{u}}=\frac{1}{Q_{d}}+\frac{1}{Q_{c}}
$$

where $Q_{d}$ is the quality factor associated with the dielectric losses and $Q_{c}$ is the quality factor associated with the wall losses. The needed quality factor to extract the complex permittivity is $Q_{d}$. $Q_{u}$ is measured with the liquid sample and $Q_{c}$ with the empty cavity. The tube holder influence is negligible in the measure of loss factor because quartz has very low losses $\left(<10^{-4}\right)$, which are completely hidden by the liquid losses. In fact, as said above, the quartz tube is used to improve the quality factor in the same manner that in [12].

It is important to remark that the theoretical modeling described in section II considers perfect electric walls (PEC). However, the real cavity has finite conductivity, which affects the measurement of the resonant frequency. For a given resonant mode, the resonant frequency shift due to the finite conductive of the cavity walls can be estimated by the perturbational expression given in [39]:

$$
\frac{f_{r}-f_{r, \text { lossless }}}{f_{r, \text { lossless }}} \cong \frac{-1}{2 Q_{c}}
$$

where $f_{r}$ and $f_{r, \text { lossless }}$ stand for the resonant frequencies of the real (lossy) and ideal (lossless) cavities, respectively, while $Q_{c}$ is the $Q$-factor of the real cavity related to losses in the metallic walls because of their finite conductivity.

The uncertainty of the measurements has been calculated following the procedure described in [40]-[41]. It is based on the calculation of the derivative of the permittivity depending on the variable that affects the accuracy and then, the propagation of errors theory is applied to determine the uncertainty of the complex permittivity as follows:

$$
u\left(\varepsilon_{r}\right)=\sqrt{\sum_{\forall i}\left(\frac{\partial \varepsilon_{r}}{\partial x_{i}} u\left(x_{i}\right)\right)^{2}}
$$

where $x_{i}$ are the different parameters involved in the problem, such as geometric dimensions, whose uncertainty $u\left(x_{i}\right)$ is given in table II; and $f_{u}$ and $Q_{u}$, whose uncertainty is given by the resolution considered in the VNA measurement, and it is around $0.5 \%$.

The complex permittivity of quartz has been obtained with experimental measurements to use an exact value at every frequency of the selected modes. In Fig. 8, the measured values of $\varepsilon_{r}^{\prime}$ and $\varepsilon_{r}^{\prime \prime}$ for $\mathrm{TM}_{011}$ and $\mathrm{EH}_{110}$ modes are shown. The obtained values agree with those provided in the literature such as in [15] or [42]. 


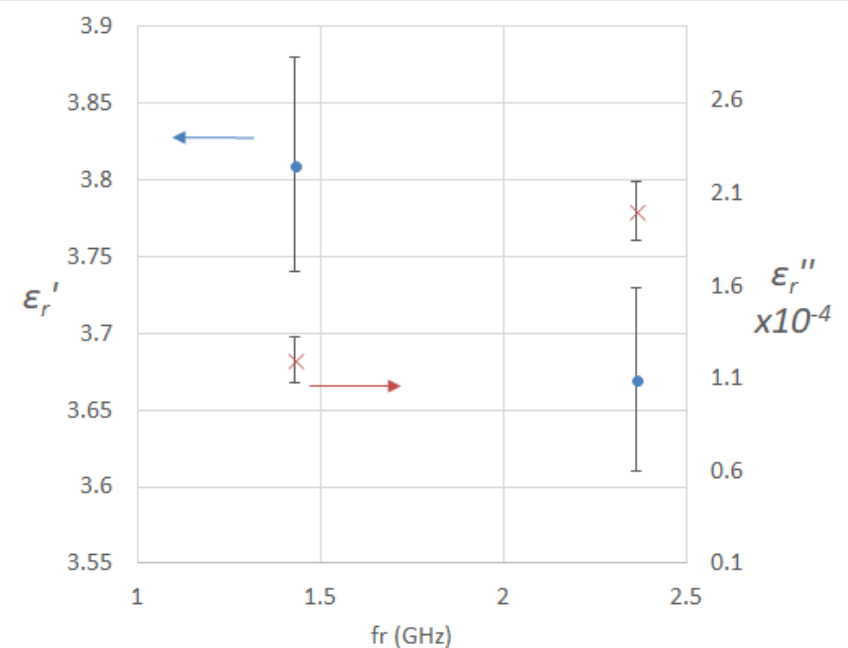

\begin{tabular}{|c|c|c|}
\hline Mode & $f_{u}(\mathrm{GHz})$ & $Q_{u}$ \\
\hline $\mathrm{TM}_{011}$ & 1.438473 & 6601.4 \\
\hline $\mathrm{EH}_{110}$ & 2.360373 & 11044 \\
\hline
\end{tabular}

Fig. 8. Measured dielectric properties of quartz holder. $Q_{c-\text { TM011 }}=6617.37, Q_{c-}$ EH110 $=10978.9$.

In table $\mathrm{V}$, measurements of the complex permittivity of reference liquids are calculated at some different frequencies corresponding with the $\mathrm{TM}_{011}$ and $\mathrm{EH}_{110}$ modes. The unloaded resonant frequency and quality factor are provided, as well as $Q_{c}$. An example of a lower loss liquid (acetone) is also included to demonstrate that the method can be successfully applied also in these cases, confirming the wide range of applicability. The obtained results are compared with reference values tabulated in [43], and the good agreement between them shows the correct behavior of the method.

\section{CONCLUSION}

In this paper, a novel dielectrometer cell has been presented and analyzed with a full-wave modal method, which takes into account all the possible set of modes. The used holder, unlike those of the literature, has quite large diameter to facilitate the pouring of the liquid.

The convergence of the method has been studied deeply and a fitting procedure has been presented in order to provide an asymptotic solution of the problem taking into account infinite number of terms in the modal expansions of the problem's formulation.

The method has been calibrated calculating the effective dimensions of the cavity through the measurements of the resonant frequency with the empty cavity. With this calibration, the error between simulations and measurements is minimized.

Some considerations have been discussed for choosing the most appropriate resonant modes to measure the complex permittivity of high loss liquids. Comparisons between the obtained permittivity of some liquids and the reference values of the literature validate the method experimentally.

The proposed cavity has been designed with fixed dimensions and the resonant frequencies cannot be tuned. However, in future works, the system could be implemented for measuring complex permittivity at some desirable frequencies, since it would improve the range of applicability of the proposed cell. This could be implemented designing a tunable cavity, where the metallic post would be movable and consequently, the resonant frequency for measuring permittivity could be tuned by the user.

\section{REFERENCES}

[1] R. N. Clarke et al., "A Guide to the characterization of dielectric materials at RF and microwave Frequencies", Institute of Measurement and Control (IMC) and The National Physical Laboratory (NPL), London, UK, PDB:3657, 2003.

[2] U. Kaatze and Y. Feldman, "Broadband dielectric spectrometry of liquids and biosystems,” Meas. Sci. Technol., vol. 17, no. 2, pp. R17R35, Dec. 2006.

[3] A. Gregory and R. Clarke, "A review of RF and microwave techniques for dielectric measurements on polar liquids," IEEE Trans. Dielectr. Electr. Insul., vol. 13, no. 4, pp. 727-743, Aug. 2006.

[4] A. Nyshadham, C. L. Sibbald, and S. S. Stuchly, "Permittivity measurements using open-ended sensors and reference liquid calibration-an uncertainty analysis," IEEE Trans. Microw. Theory Techn., vol. 40, no. 2, pp. 305-314, Feb 1992.

[5] J. Baker-Jarvis, M. D. Janezic and C. A. Jones, "Shielded open-circuited sample holder for dielectric measurements of solids and liquids," IEEE Trans. Instrum. Meas., vol. 47, no. 2, pp. 338-344, Apr 1998.

[6] J. Baker-Jarvis et al., "Measuring the permittivity and permeability of lossy materials: Solids liquids metals building materials and negative index materials,” NIST Tech. Note 1536, Boulder, CO, USA, Feb. 2005.

[7] S. Mueller et al., "Broad-band microwave characterization of liquid crystals using a temperature-controlled coaxial transmission line," IEEE Trans. Microw. Theory Techn., vol. 53, no. 6, pp. 1937-1945, June 2005.

[8] A. H. Sklavounos and N. S. Barker, "Liquid-Permittivity Measurements Using a Rigorously Modeled Overmoded Cavity Resonator,” IEEE Trans. Microw. Theory Techn., vol. 62, no. 6, pp. 1363-1372, June 2014.

[9] K. B. Yu, S. G. Ogourtsov, V. G. Belenky, A. B. Maslenikov, and A. S. Omar, "Accurate microwave resonant method for complex permittivity

TABLE V

MEASUREMENTS OF THE COMPLEX PERMITTIVITY OF SOME REFERENCE LIQUIDS WITH TM $\mathrm{M}_{011}$ AND EH $_{110}$ MODES. $^{\circ}$ $\mathrm{Q}_{\text {с-тм011 }}=6617.37, \mathrm{Q}_{\text {с-ен110 }}=10978.9$, TEMPERATURE $25^{\circ} \mathrm{C}$.

\begin{tabular}{|c|c|c|c|c|c|c|l|}
\hline Material & Mode & $f_{u}(\mathrm{GHz})$ & $Q_{u}$ & $\varepsilon_{r}^{\prime} \pm u\left(\varepsilon_{r}^{\prime}\right)$ & $\varepsilon_{r}^{\prime \prime} \pm u\left(\varepsilon_{r}^{\prime \prime}\right)$ & $\begin{array}{c}\varepsilon_{r}^{\prime} \pm u\left(\varepsilon_{r}^{\prime}\right) \\
{[43]}\end{array}$ & $\begin{array}{c}\varepsilon_{r}^{\prime \prime} \pm u\left(\varepsilon_{r}^{\prime \prime}\right) \\
{[43]}\end{array}$ \\
\hline \multirow{2}{*}{$\begin{array}{c}\text { Deionized } \\
\text { Water }\end{array}$} & $\mathrm{TM}_{011}$ & 1.4150465 & 1276.5 & $77.96 \pm 0.09$ & $5.56 \pm 0.04$ & $77.8 \pm 0.08$ & $5.25 \pm 0.02$ \\
\cline { 2 - 8 } & $\mathrm{EH}_{110}$ & 2.2789647 & 34.51 & $76.60 \pm 0.05$ & $10.78 \pm 0.04$ & $77.2 \pm 0.06$ & $8.38 \pm 0.03$ \\
\hline \multirow{2}{*}{ Methanol } & $\mathrm{TM}_{011}$ & 1.4218175 & 242.77 & $28.52 \pm 0.06$ & $10.98 \pm 0.04$ & $28.05 \pm 0.03$ & $10.18 \pm 0.02$ \\
\cline { 2 - 8 } & $\mathrm{EH}_{110}$ & 2.3533687 & 186.06 & $21.78 \pm 0.07$ & $11.8 \pm 0.05$ & $22.91 \pm 0.04$ & $13.01 \pm 0.03$ \\
\hline \multirow{2}{*}{$\begin{array}{c}\text { Dimethyl } \\
\text { sulfoxide }\end{array}$} & $\mathrm{TM}_{011}$ & 1.4187409 & 593.71 & $45.51 \pm 0.04$ & $6.95 \pm 0.03$ & $45.36 \pm 0.03$ & $6.62 \pm 0.01$ \\
\cline { 2 - 8 } & $\mathrm{EH}_{110}$ & 2.3405594 & 123.86 & $41.27 \pm 0.06$ & $11.5 \pm 0.03$ & $43.56 \pm 0.04$ & $10.42 \pm 0.02$ \\
\hline \multirow{2}{*}{ Acetone } & $\mathrm{TM}_{011}$ & 1.4246041 & 2299.8 & $21.34 \pm 0.05$ & $0.61 \pm 0.01$ & $21.18 \pm 0.03$ & $0.58 \pm 0.008$ \\
\cline { 2 - 8 } & $\mathrm{EH}_{110}$ & 2.3529096 & 2192 & $21.76 \pm 0.04$ & $1.2 \pm 0.02$ & $21.15 \pm 0.03$ & $0.95 \pm 0.005$ \\
\hline
\end{tabular}


measurements of liquids [biological]," IEEE Trans. Microw. Theory Techn., vol. 48, no. 11, pp. 2159-2164, Nov 2000.

[10] U. Raveendranath, S. Bijukumar, and K. T. Mathew, "Broadband coaxial cavity resonator for complex permittivity measurements of liquids," IEEE Trans. Instrum. Meas, vol. 49, no. 6, pp. 1305-1312, Dec 2000.

[11] H. Kawabata, H. Tanpo, and Y. Kobayashi, "A rigorous analysis of a TM010 mode cylindrical cavity to measure accurate complex permittivity of liquid,” in 33rd Eur. Microw. Conf., Munich, Germany, 2003, pp. 759-762.

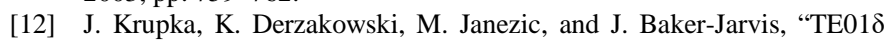
Dielectric-resonator technique for precise measurements of the complex permittivity of lossy liquids at frequencies below $1 \mathrm{GHz}$," Precision Electromagn. Meas. Conf. Dig., London, UK, 2004, pp. 469-470.

[13] P. Kanpan, E. Khansalee, N. Puangngernmak, and S. Chalermwisutkul, "TM010 mode cylindrical cavity for complex permittivity measurement of liquid using field analysis technique," 2012 9th ECTI-CON, Phetchaburi, 2012, pp. 1-4.

[14] A. Stefanski and J. Krupka, "Complex permittivity measurements of lossy liquids at microwave frequencies," 17th Int. IEEE Microw., Radar, Wireless Commun. Conf., Wroclaw, Poland, 2008, pp. 1-4.

[15] J. Krupka, "Measurements of the complex permittivity of highly concentrated aqueous $\mathrm{NaCl}$ solutions and ferrofluid employing microwave cylindrical cavities,” Meas. Sci. Technol., 26 095702, Jul. 2015.

[16] Z. E. Eremenko, E. M. Ganapolskii, and V. V. Vasilchenko, "Exact calculated resonator method for permittivity measurement of high loss liquids at millimetre wavelength,” Meas. Sci. Technol., vol. 16, pp. 1619-1627, Jul. 2005.

[17] N. Meyne, G. Fuge, A. P. Zeng, and A. F. Jacob, "Resonant Microwave Sensors for Picoliter Liquid Characterization and Nondestructive Detection of Single Biological Cells," IEEE Journ. of Electromagn., RF and Microw. in Medic. and Bio,, vol. 1, no. 2, pp. 98-104, Dec. 2017.

[18] J. C. Booth, N. D. Orloff, J. Mateu, M. Janezic, M. Rinehart, and J. A. Beall, "Quantitative Permittivity Measurements of Nanoliter Liquid Volumes in Microfluidic Channels to $40 \mathrm{GHz}$," IEEE Trans. Instrum. Meas., vol. 59, no. 12, pp. 3279-3288, Dec. 2010.

[19] A. A. Abduljabar, D. J. Rowe, A. Porch and D. A. Barrow, "Novel Microwave Microfluidic Sensor Using a Microstrip Split-Ring Resonator," IEEE Trans. Microw. Theory Techn., vol. 62, no. 3, pp. 679688, Mar. 2014.

[20] P. Vélez, L. Su, K. Grenier, J. Mata-Contreras, D. Dubuc, and F. Martín, "Microwave Microfluidic Sensor Based on a Microstrip Splitter/Combiner Configuration and Split Ring Resonators (SRRs) for Dielectric Characterization of Liquids," IEEE Sensors J., vol. 17, no. 20, pp. 6589-6598, Oct. 2017.

[21] A. A. Abduljabar, N. Clark, J. Lees, and A. Porch, "Dual Mode Microwave Microfluidic Sensor for Temperature Variant Liquid Characterization," IEEE Trans. Microw. Theory Techn, vol. 65, no. 7, pp. 2572-2582, Jul. 2017.

[22] J. Blackburn, "Solving the double eigenvalue problem: A study of mode matching in arbitrary layer dielectric resonators," IEE Proc. - Microw., Antennas and Propag., vol. 153, no. 5, pp. 447-455, Oct. 2006.

[23] F. L. Penaranda-Foix and J. M. Catala-Civera, "Circuital analysis of cylindrical structures applied to the electromagnetic resolution of resonant cavities," Passive Microwave Components and Antennas, 1st ed. Rijeka, Croatia: IN-TECH, Apr. 2010, ch. 7. [Online]. Available: Components-and-Antennas.

[24] F. L. Penaranda-Foix, M. D. Janezic, J. M. Catala-Civera, and A. J. Canos, "Full-Wave Analysis of Dielectric-Loaded Cylindrical Waveguides and Cavities Using a New Four-Port Ring Network," IEEE Trans. Microw. Theory Techn., vol. 60, no. 9, pp. 2730-2740, Sept. 2012.

[25] D. Marqués-Villarroya, F. Peñaranda-Foix, B. García-Baños, and A.J. Canós, "Measurement of complex permittivity using azimuthal modes in a split cavity,” Ampere 2017 Conf., Delf, Netherland, Sept. 2017.

[26] D. Marques-Villarroya, F. Penaranda-Foix, B. Garcia-Banos, J. M. Catala-Civera and J. Daniel Gutierrez-Cano, "Analysis of an overmoded re-entrant cavity,” 2017 47th Eur. Microw. Conf., Nuremberg, Germany, 2017, pp. 440-443.

[27] D. Marqués-Villarroya, A.J. Canos, F. Peñaranda-Foix, B. GarcíaBaños, and J. M. Catalá-Civera, "Determination of the Complex Permittivity of High Loss Liquids with a Novel Reentrant Cavity,” 2018 IEEE MTT-S Int. Microw. Symp. Dig., Philadelphia, PA, 2018, pp. 1-4.

[28] D. Marques-Villarroya, F. Penaranda-Foix, B. Garcia-Banos, and J. M. Catala-Civera, "Extended Full-Wave Analysis of a Multimode Circular
Cavity with Insertion Hole,” 2018 48th Eur. Microw. Conf., Madrid, Spain, Sept. 2018, pp. 1-4.

[29] K. Zhang and D. Li, Electromagnetic Theory for Microwave and Optoelectronics, New York, NY, USA: Springer-Verlag, 2008, ch.4.10

[30] W.C. Chew, Lectures on Theory of Microwave and Optical Waveguides, 2012, [Online] Available: http://wcchew.ece.illinois.edu/chew/course/tgwAll20121211.pdf , Dec. 2012, ch. 5.2.1.

[31] C. A. Balanis, Advanced Engineering Electromagnetics, Hoboken, NJ, USA: John Wiley \& Sons, 1989.

[32] R. Mittra, "Relative convergence of the solution of a doubly infinite set of equations,” J. Res. Nat. Bur. Stand., vol. 67D, no. 2, pp. 245-254, Mar./Apr. 1963.

[33] P. H. Masterman and P.J.B. Clarricoats, "Computer field-matching solution of waveguide transverse discontinuities", Proc. IET., vol. 118, no. 1. Jan. 1971, pp. 51-63.

[34] R. Sorrentino, M. Mongiardo, F. Alessandri, and G. Schiavon, "An Investigation of the numerical properties of the mode-matching technique”. Int. Journ. of Numeric. Model., vol. 4., pp. 19-43, Apr. 1991.

[35] O. P. Franza and W. C. Chew, "Recursive mode matching method for multiple waveguide junction modeling," IEEE Trans. Microw. Theory Techn., vol. 44, no. 1, pp. 87-92, Jan 1996.

[36] J. C. Lagarias, J. A. Reeds, M. H. Wright, and P. E. Wright. "Convergence Properties of the Nelder-Mead Simplex Method in Low Dimensions.” SIAM Journal of Optimization, vol. 9, no. 1, pp. 112-147, 1998.

[37] A. J. Canós, J. M. Catalá-Civera, F. L. Peñaranda-Foix, and E. de los Reyes Davó, "A novel technique for deembeding the unloaded resonance frequency from measurements of microwave cavities," IEEE Trans. Microw. Theory Techn., vol. 54, no. 8, pp. 3407-3416, Aug. 2006.

[38] D. Kajfez, Q Factor, Oxford, MS, USA: Vector Fields, 1994.

[39] R. F. Harrington, Time-Harmonic Electromagnetic Fields, 2nd Edition: Wiley, IEEE Press, pp. 372, 2001.

[40] J. Baker-Jarvis, M.D. Janezic, P.D. Domich, and R.G. Geyer, “Analysis of an Open-Ended Coaxial Probe with Lift-Off for Nondestructive Testing”, IEEE Trans. Instrum. Meas., vol. 43, no 5, Oct., pp. 711-718, Oct. 1994.

[41] S. Bell, Beginner's Guide to Uncertainty of Measurement (Issue 2), National Physical Laboratory (NPL), ISSN 1368-6550. August 1999, Issue 2 with amendments March 2001.

[42] R. von Hippel, Dielectric materials and applications, The M.I.T. press, 1954.

[43] A. Gregory and R. N. Clarke, "Tables of the complex permittivity of dielectric reference liquids at frequencies up to $5 \mathrm{GHz}$ ", Material Division of The National Physical Laboratory (NPL), UK, NPL Report MAT 23, Jan. 2012. 